PROCEEDINGS OF THE

AMERICAN MATHEMATICAL SOCIETY

Volume 128, Number 6, Pages 1853-1857

S 0002-9939(99)05250-8

Article electronically published on September 30, 1999

\title{
AN INDUCTIVE EXPLICIT CONSTRUCTION OF *-PRODUCTS ON SOME POISSON MANIFOLDS
}

\author{
SANTOS ASIN LARES
}

(Communicated by Peter Li)

\begin{abstract}
We extend the Cahen Gutt coboundary construction on cotangent bundles of $n$-dimensional parallelisable manifolds to manifolds which admit $n$ global vector fields defining a parallelisation on a dense open set. This result is used to give an inductive explicit construction of $*$-products on certain Poisson manifolds.
\end{abstract}

\section{INTRODUCTION}

The theory of formal deformation quantization of Poisson manifolds was introduced by Bayen et al. in [2. The main notion of this theory is the concept of a *-product. The general question of the existence of such a product for symplectic manifolds has been completely solved by several authors, using various techniques [5], [14, [11. Recently, M. Kontsevitch has proved the existence of $*$-products on arbitrary finite-dimensional Poisson manifolds [9]. Nevertheless, since Kontsevitch's result is not given by a simple geometrical construction, it has increased the interest of having a simple geometrical of $*$-products on non-regular Poisson manifolds.

Since every Poisson manifold splits into a collection of symplectic submanifolds, known as the leaves of the symplectic foliation, one naturally asks whether a *product on a Poisson manifold restricts to give a $*$-product on the symplectic leaves. Lately, in [4, 13] it has been proved that such $*$-products do not always exist. When they exist we called them tangential. In particular, the dual of the so-called "book algebra" with the Lie Poisson structure admits a tangential *-product [1]. Furthermore, this example provides the basic idea to construct explicit $*$-products on some other Poisson manifolds (see Theorem 1).

The goal of this paper will be to give such a construction. In order to do this, we first generalize a coboundary construction due to Cahen and Gutt [3], and use this result to construct explicitly the $*$-product.

Using a different method a similar result has been obtained in [7]. However, the approach used there does not provide an explicit construction of a $*$-product.

Received by the editors July 21, 1998.

1991 Mathematics Subject Classification. Primary 58F06, 53Z05; Secondary 81Q99.

Key words and phrases. *-product, deformation quantization.

The author was supported by a grant from the University of Warwick.

(C)2000 American Mathematical Society 
A Poisson structure on a manifold $M$ is a Lie algebra structure $\{\cdot, \cdot\}$ on $C^{\infty}(M)$ which satisfies the derivation property

$$
\{f g, h\}=f\{g, h\}+\{f, h\} g, \quad \forall f, g, h \in C^{\infty}(M) .
$$

The operation $\{\cdot, \cdot\}$ determines a contravariant skew-symmetric 2-tensor $\Lambda$ such that $\{f, g\}=\Lambda(d f, d g)$. A Poisson structure may also be defined by such a tensor (the Poisson tensor); the Jacobi identity for the Poisson structure is equivalent to the vanishing of the so-called Schouten bracket $[\Lambda, \Lambda]_{s}=0$ (see [2]).

If $(M, \Lambda)$ is a Poisson manifold we set $N=C^{\infty}(M)$. Let $N[[\lambda]]$ be the space of formal power series in a parameter $\lambda$, with coefficients in $N$.

Definition 1 ([2]). A *-product on $(M, \Lambda)$ is a bilinear map $N^{2} \longrightarrow N[[\lambda]]$ defined by

$$
(f, g) \longrightarrow f * g=\sum_{n=0}^{\infty} \lambda^{n} C_{n}(f, g),
$$

where the so-called cochains $C_{n}$, are bilinear maps with values in $N$ and satisfy the following axioms:

1. $C_{0}(f, g)=f g, \quad C_{1}(f, g)=\{f, g\}, \quad \forall f, g \in C^{\infty}(M)$;

2. $C_{n}(f, g)=(-1)^{n} C_{n}(g, f), \quad \forall f, g \in C^{\infty}(M), \quad \forall n \geq 1$;

3. $C_{n}(f, k)=0, \quad \forall f \in C^{\infty}(M), \quad \forall k \in \mathbb{R}, \quad \forall n \geq 1$;

4. $\sum_{r+s=k} C_{r}\left(C_{s}(f, g), h\right)=\sum_{r+s=k} C_{r}\left(f, C_{s}(g, h)\right), k \geq 0$.

The theory of deformations in the sense of [6] relates the deformations of an associative algebra to the corresponding Hochschild cohomology.

Definition 2. A (Hochschild) $p$-cochain is a $p$-linear map $N^{p} \rightarrow N$. The Hochschild coboundary of a $p$-cochain is the $(p+1)$-cochain $\partial C$ given by

$$
\begin{gathered}
\partial C\left(u_{0}, \ldots, u_{p}\right)=u_{0} C\left(u_{1}, \ldots, u_{p}\right)-C\left(u_{0} u_{1}, u_{2}, \ldots, u_{p}\right)+C\left(u_{0}, u_{1} u_{2}, \ldots, u_{p}\right) \\
+\cdots+(-1)^{p} C\left(u_{0}, u_{1}, \ldots, u_{p-1} u_{p}\right)+(-1)^{p+1} C\left(u_{0}, \ldots, u_{p-1}\right) u_{p} .
\end{gathered}
$$

A cochain $C$ is called differential if it is defined by multi-differential operators in each argument. A *-product is called differential if all its cochains are differential. In [12] it has been proved that if $E$ is a $p$-cocycle (differential and null on the constants), then there exist a skew-symmetric contravariant smooth $p$-tensor $A$ and a $(p-1)$-cochain $C$ such that

$$
E\left(f_{1}, \ldots, f_{p}\right)=\partial C\left(f_{1}, \ldots, f_{p}\right)+A\left(d f_{1}, \ldots, d f_{p}\right), \quad f_{i} \in C^{\infty}(M) .
$$

A bilinear map (1) is said to be an associative formal deformation up to the order $k$ if

$$
(f * g) * h-f *(g * h)=0, \quad f, g, h \in C^{\infty}(M),
$$

is satisfied modulo $\lambda^{k+1}$. By developing (3) into powers of $\lambda$, the coefficients of $\lambda^{t}$ will vanish if

$$
E_{t}(f, g, h):=\sum_{r+s=t, r, s \geq 1} C_{r}\left(C_{s}(f, g), h\right)-C_{r}\left(f, C_{s}(g, h)\right)=\partial C_{t}(f, g, h) .
$$

Thus, an associative formal deformation up to the order $k$ can be extended to one of order $k+1$ provided that the cocycle $E_{k+1}$ is a 3 -coboundary. 


\section{TANGENTIAL $*$-PRODUCTS}

Let $(M, \Lambda)$ be a Poisson manifold, and let $O$ be a symplectic leaf.

Definition 3. Let $x \in O$. A differential operator $D$ on $M$ is tangential to $O$ at $x$, if there exist a neighbourhood $V$ of $x$ in $O$ and a neighbourhood $U$ of $V$ in $M$, such that when $\varphi_{1}, \varphi_{2} \in C^{\infty}(U)$ with $\varphi_{\left.1\right|_{V}}=\varphi_{\left.2\right|_{V}}$, then

$$
D\left(\varphi_{1}\right)_{\left.\right|_{V}}=D\left(\varphi_{2}\right)_{\left.\right|_{V}} .
$$

A bi-differential operator $C$ on $M$ is said to be tangential to $O$, if for any function $f \in C^{\infty}(M)$, the differential operators $C(f, \cdot)$ and $C(\cdot, f)$ are tangential to $O$, at $x$ for all $x \in O$.

Definition 4. A differential *-product is called tangential to $O$, if all its cochains $C_{n}, n \geq 1$, are tangential.

\section{A COBOUNDARY CONSTRUCTION}

In what follows, we shall use the summation convention on pairs of upper and lower indices. Let $(M, \Lambda)$ be a Poisson manifold of dimension $n$, and let $T^{1}, \ldots, T^{n}$ be smooth vector fields on $M$ such that they are pointwise linearly independent on a dense open set of $M$. The following proposition is a simple generalization of Proposition 2 in 3 . The argument given in 3 is combinatorial, and is based on 3 lemmas which in fact only require independence of the vector fields $T^{i}$ on a dense open set.

Proposition 1 ([3]). Let $E$ be a differential 3-cocycle (null on the constants), of the form

$$
E(f, g, h)=\sum_{0<a, b, c \leq K} E_{i_{1} \ldots i_{a}, j_{1} \ldots j_{b}, k_{1} \ldots k_{c}} T^{i_{1}} \cdots T^{i_{a}} f T^{j_{1}} \cdots T^{j_{b}} g T^{k_{1}} \cdots T^{k_{c}} h,
$$

where $f, g, h \in C^{\infty}(M)$, and $E_{i_{1} \ldots i_{a}, j_{1} \ldots j_{b}, k_{1} \ldots k_{c}}$ are smooth functions on $M$ symmetric in the $i$ 's, in the $j$ 's and in the $k$ 's. Then, there is a 2-cochain $C$ completely determined by $E$ of the form

$$
C(f, g)=\sum_{0<p, q \leq K} C_{i_{1} \ldots i_{p}, j_{1} \ldots j_{q}} T^{i_{1}} \cdots T^{i_{p}} f T^{j_{1}} \cdots T^{j_{q}} g
$$

such that $E=\partial C+A$, where $A$ is the completely antisymmetric part of $E$, i.e., a 3-contravariant smooth tensor. Moreover, the coefficients $C_{i_{1} \ldots i_{p}, j_{1} \ldots j_{q}}$ are constant (rational) linear combinations of the coefficients $E_{k_{1} \ldots k_{a}, l_{1} \ldots l_{b}, m_{1} \ldots m_{c}}$ of $E$.

Remark 1 . Note that if the $C_{r}$ 's $(r \leq k)$ satisfy the symmetry properties of Definition 1, then the cocycle $E_{k+1}$ satisfies $E_{k+1}(f, g, h)=(-1)^{k} E_{k+1}(h, g, f)$. Thus, if $E_{k+1}=\partial C_{k+1}$ we can always assume that $C_{k+1}(f, g)=(-1)^{k+1} C_{k+1}(g, f)$ just by replacing $C_{k+1}$ by its symmetrization or antisymmetrization.

Theorem 1. Let $(M, \Lambda)$ be a Poisson manifold, and let us assume that there exist $T^{1}, T^{2}$ smooth vector fields on $M$ such that they are pointwise linearly independent on a dense open set of $M$, and such that $\Lambda$ can be written as $\Lambda=T^{1} \wedge T^{2}$. Then, there is a *-product on $(M, \Lambda)$ with 2-cochains $C_{r}$ of the form (51). 
Proof. By assumption the Poisson structure $C_{1}$ on $M$ admits the expression $C_{1}(f, g)$ $=T^{1} f T^{2} g-T^{2} f T^{1} g$. Let us assume that there exist $k(k \geq 1) 2$-cochains $C_{1}, \ldots, C_{k}$ constructed recursively (using Proposition \) from the equations

$$
E_{t}=\partial C_{t} \quad t=1, \ldots, k,
$$

defining a deformation (up to the order $k$ ) on $M$ so that

$$
C_{t}(f, g)=\sum_{0<p, q \leq K_{t}} C_{i_{1} \ldots i_{p}, j_{1} \ldots j_{q}} T^{i_{1}} \cdots T^{i_{p}} f T^{j_{1}} \cdots T^{j_{q}} g
$$

where all the $T^{i}$ 's and $T^{j}$ 's are $T^{1}$ or $T^{2}$.

Since $\left[T^{1} \wedge T^{2}, T^{1} \wedge T^{2}\right]_{s}=0$, it follows that $\left[T^{1}, T^{2}\right]_{s}=f_{1} T^{1}+f_{2} T^{2}\left(f_{1}, f_{2} \in\right.$ $C^{\infty}(M)$ ), and so that $E_{k+1}$ expressed as in Proposition 1 only includes $T^{1}$ 's and $T^{2}$ 's. Let $C_{k+1}$ and $A_{k+1}$ be the 2-cochains constructed by means of Proposition 1 such that $E_{k+1}=\partial C_{k+1}+A_{k+1}$. Then, since $A_{k+1}$ is a 3 -contravariant (skewsymmetric) tensor only including $T^{1}$ and $T^{2}$, it follows that $A_{k+1}$ vanishes, i.e., $E_{k+1}=\partial C_{k+1}$. Thus, the theorem follows by induction on $k$.

Remark 2. The $*$-product constructed is tangential to the 2-dimensional symplectic leaves.

\section{EXAMPLES}

Example 1. Let $\mathfrak{g}$ be the Lie algebra (book algebra) with basis $\left(e_{1}, e_{2}, e_{3}\right)$, such that $\left[e_{1}, e_{2}\right]=0,\left[e_{1}, e_{3}\right]=e_{1},\left[e_{2}, e_{3}\right]=e_{2}$. Let $\left(x_{1}, x_{2}, x_{3}\right)$ be a coordinate system on $\mathfrak{g}^{*}$ determined by the dual basis $\left(e^{1}, e^{2}, e^{3}\right)$. The Lie-Poisson structure $\Lambda$ can be expressed in terms of the above global coordinate system as

$$
\Lambda=\left(x_{1} \frac{\partial}{\partial x_{1}}+x_{2} \frac{\partial}{\partial x_{2}}\right) \wedge \frac{\partial}{\partial x_{3}} .
$$

Therefore, using Theorem 1 we get an explicit *-product (in fact, the Gutt *-product [8]).

Example 2. Let us consider the Lie group $S U(2)$, and let us choose

$$
e_{2}:=\left(\begin{array}{cc}
i & 0 \\
0 & -i
\end{array}\right), \quad e_{3}:=\left(\begin{array}{cc}
0 & 1 \\
-1 & 0
\end{array}\right), \quad e_{4}:=\left(\begin{array}{cc}
0 & i \\
i & 0
\end{array}\right)
$$

as a basis of its Lie algebra $\mathfrak{s u}(2)$.

Let $S p(1)$ be the group of unitary quaternions. We identify $S p(1)$ and $S U(2)$ as Lie groups by means of

$$
\begin{gathered}
\psi: S p(1) \longrightarrow S U(2) \\
\left(x_{1}, x_{2}, x_{3}, x_{4}\right) \longrightarrow\left(\begin{array}{cc}
x_{1}+x_{2} i & x_{3}+x_{4} i \\
-x_{3}+x_{4} i & x_{1}-x_{2} i
\end{array}\right) .
\end{gathered}
$$

As usual, we denote by $R_{g}\left(L_{g}\right)$ the right translation (left translation) map. Let $\mathbf{r}=e_{3} \wedge e_{4} \in \bigwedge^{2} \mathfrak{s u}(2)$; then the Iwasawa-Poisson-Lie structure $\pi$ on $S U(2)$ is defined by [10]

$$
\pi(g):=d R_{g} \mathbf{r}-d L_{g} \mathbf{r}, \quad g \in S U(2)
$$


The linearization (at the identity) of this Poisson structure is isomorphic to the book algebra, and therefore this Poisson structure can be considered as the nonlinear version of that in Example 1. Let $X_{i}(i=2,3,4)$ be the right invariant vector fields on $S U(2)$ corresponding to $e_{i}$. We define two vector fields on $S U(2)$ by setting

$$
\begin{gathered}
T^{1}:=x_{2} X_{2}+x_{3} X_{3}+x_{4} X_{4}, \\
T^{2}:=\left(-2 x_{1}\right) X_{2}+\left(-2 x_{4}\right) X_{3}+\left(2 x_{3}\right) X_{4} .
\end{gathered}
$$

A straightforward computation shows that the Poisson structure $\pi$ on $S U(2)$ can be written as $\pi=T^{1} \wedge T^{2}$. Hence, using Theorem 1 one can construct an explicit *-product on this Poisson manifold (the non-linear version of the one constructed in the previous example).

\section{ACKNOWLEDGMENTS}

The author would like to deeply thank J. Rawnsley for his invaluable help throughout this work, S. Gutt for helpful comments on the manuscript, and V. Chloup-Arnould and J. L. Cisneros for important remarks and their help in the organization of the paper.

\section{REFERENCES}

[1] S. Asin. On tangential properties of the Gutt *-product. Journal of Geometry and Physics, 24:164-172, 1988. MR 98i:58108

[2] F. Bayen, M. Flato, C. Fronsdal, A. Lichnerowicz, and D. Sternheimer. Deformation theory and quantization I and II. Ann. Physics, 111(61-151), 1978. MR 58:14737a

[3] M. Cahen and S. Gutt. Regular *-representations of Lie algebras. Lett. in Math. Physics, 6:395-404, 1982.

[4] M. Cahen, S. Gutt, and J.H. Rawnsley. On tangential star products for the coadjoint Poisson structure. Comm. Math. Physics, 180:99-108, 1996. MR 97e:58101

[5] B. Fedosov. A simple geometrical construction of deformation quantization. J. Diff Geom., 40:213-238, 1994. MR 95h:58062

[6] M. Gerstenhaber. On the deformation of rings and algebras. Ann. of Math., 79:59-103, 1964. MR 30:2034

[7] J. Grabowski. Hochschild cohomology and quantization of Poisson structures. Rend. Circ. Matem. di Palermo, 2(Suppl. no 37):87-91, 1994. MR 96f:58068

[8] S. Gutt. An explicit *-product on the cotangent bundle of a Lie group. Lett. Math. Phys., 7:249-258, 1983. MR 85g:58037

[9] M. Kontsevich. Deformation quantization of Poisson manifolds I. q-alg 9709040, 1997.

[10] J.H. Lu and A. Weinstein. Poisson-Lie groups, dressing transformations and Bruhat decompositions. J. Diff Geom., 31:501-526, 1990. MR 91c:22012

[11] H. Omori, Y. Maeda, and A. Yoshioka. Weyl manifolds and deformation quantization. Advances in Math., 85:224-255, 1991. MR 92d:58071

[12] J. Vey. Deformation du crochet de Poisson sur une varieté symplectique. Commentarii Math. Helvet., 50:421-454, 1975. MR 54:8765

[13] A. Weinstein. Tangential deformation quantization and polarized symplectic groupoids. In D. Sternheimer et al., editor, Deformation theory and symplectic geometry, volume 20 of Mathematical Physics Studies, pages 301-327. Kluwer, 1997. CMP 98:04

[14] M. De Wilde and P. Lecomte. Existence of star-products and of formal deformations of the Poisson Lie algebra of arbitrary symplectic manifolds. Lett. in Math. Physics, 7:487-496, 1983. MR 85j:17021

Mathematical Institute, University of Warwick, CV4-7AL, United Kingdom

E-mail address: asin@maths.warwick.ac.uk 